\title{
Capacidade funcional, desempenho e solicitação metabólica em futebolistas profissionais durante situação real de jogo monitorados por análise cinematográfica*
}

\author{
Glydiston Egberto Oliveira Ananias ${ }^{1}$, Eduardo Kokubun ${ }^{2}$, Renato Molina ${ }^{3}$, \\ Paulo Roberto Santos Silva ${ }^{4}$ José Roberto Cordeiro ${ }^{5}$ \\ Instituto de Biociências da Universidade Estadual Paulista (Unesp) e Seção de Fisiologia \\ do Exercício do Departamento Médico da Associação Portuguesa de Desportos, SP, Brasil
}

\section{RESUMO}

Foi objetivo deste estudo caracterizar a relação entre o nível de aptidão física, desempenho e solicitação metabólica em futebolistas durante situação real de jogo. Seis jogadores de futebol profissional com média de idade de 20,8 $\pm 2,6$ anos (17-25), peso 70,4 $\pm 7,5 \mathrm{~kg}(63-81,3)$ e altura $173,3 \pm$ 9,7cm (166-188), foram submetidos a testes de aptidão física em campo e análise cinematográfica durante a partida.

Os testes de aptidão física foram realizados em campo, com medições de lactato sanguíneo. A via metabólica alática foi avaliada por meio de cinco corridas na distância de $30 \mathrm{~m}$, em velocidade máxima, com pausa passiva de um minuto entre cada corrida. As concentrações de lactato foram medidas no $1 \stackrel{\circ}{\circ} 3^{\circ}$ e $5^{\circ}$ m minuto após o término das cinco corridas.

Para detecção do limiar anaeróbio foram realizadas 3 corridas de $1.200 \mathrm{~m}$ nas intensidades de 80,85 e $90 \%$ da velocidade máxima para essa distância, com intervalo passivo de 15 minutos entre cada corrida. As dosagens de lactato san-

1. Fisicultor da Associação Portuguesa de Desportos.

2. Professor da Unesp.

3. Mestre em Fisiologia.

4. Fisiologista da Associação Portuguesa de Desportos.

5. Cardiologista e Vice-Presidente de Medicina da Associação Portuguesa de Desportos.

Endereço para correspondência:

Associação Portuguesa de Desportos - SP - Brasil

Departamento Médico - Seção de Fisiologia do Exercício

Glydiston Egberto Oliveira Ananias

Rua da Piscina, 33 - Canindé

03034-070 - São Paulo, SP

Tel. (011) 224-0400 - R-224/228

Fax (011) 228-8449 guíneo foram feitas no 1 으 3 으 e 5 을inuto de repouso passivo após cada corrida.

Os futebolistas foram submetidos à filmagem individual durante o transcorrer do jogo e as concentrações de lactato foram medidas antes, no intervalo e no final da partida para análise da solicitação energética e metabólica, respectivamente. Os seguintes resultados foram verificados: 1) o limiar anaeróbio em velocidade de corrida, correspondente à concentração de lactato sanguíneo de $4 \mathrm{mmol} . \mathrm{L}^{-1}$ foi encontrado aos $268 \pm 28 \mathrm{~m} \cdot \mathrm{min}^{-1}$ ou $16,1 \pm 1,6 \mathrm{~km} \cdot \mathrm{h}^{-1} ; 2$ ) a velocidade média e a concentração de lactato máximo nas corridas de $30 \mathrm{~m}$ foram de $6,9 \pm 0,2 \mathrm{~m} \cdot \mathrm{s}^{-1}$ e $4,5 \pm 1,0 \mathrm{mmol} . \mathrm{L}^{-1}$, respectivamente; 3) a distância total percorrida foi de $10.392 \pm$ $849 \mathrm{~m}$, sendo $5.446 \pm 550 \mathrm{~m}$ para o primeiro e $4.945 \pm 366 \mathrm{~m}$ para o segundo tempo, respectivamente; 4) os valores médios encontrados nas concentrações de lactato sanguíneo foram de $1,58 \pm 0,37 ; 4,5 \pm 0,42$ e $3,46 \pm 1,54 \mathrm{mmol} . \mathrm{L}^{-1}$ antes, no intervalo do primeiro para o segundo tempo e ao final da partida, respectivamente; e 5) a distância média total atingida ao final das partidas pelos jogadores de meio-campo $(10.910 \pm 121 \mathrm{~m})$ foi ligeiramente maior que a percorrida pelos atacantes $(10.377 \pm 224 \mathrm{~m})$ e defensores $(9.889 \pm 102 \mathrm{~m})$, mas não significativa. Houve correlação negativa $(r=-0,84 ; \mathrm{p}<$ $0,05)$ entre o limiar anaeróbio $\left(268 \pm 28 \mathrm{~m} \cdot \mathrm{min}^{-1}\right.$ ou $16,1 \pm$ $\left.1,6 \mathrm{~km} \cdot \mathrm{h}^{-1}\right)$ e a concentração de lactato sanguíneo $(4,5 \pm 0,42$ mmol. $\mathrm{L}^{-1}$ ) no primeiro tempo do jogo. Portanto, os resultados sugerem que a capacidade aeróbia é um determinante importante para suportar a longa duração da partida e recuperar mais rapidamente os futebolistas dos esforços realizados em alta intensidade, com o desenvolvimento de concentrações de lactato sanguíneo menores ao final do primeiro e segundo tempo das partidas.

Palavras-chave: Jogador de futebol profissional. Limiar anaeróbio. Lactato. Distância percorrida. Medicina esportiva. 


\section{ABSTRACT}

Functional capacity, performance and metabolic needs in professional soccer players during real match-play assessed by computer-video analysis

The purpose of this investigation was to caracterize the relationship between the level of physical fitness, performance and metabolic needs in soccer players during a real matchplay. Six professional soccer players aged $20.8 \pm 2.6$ years old (17-25), weight 70.4 $\pm 7.5 \mathrm{~kg}$ (63-81.3) and height 173.3 $\pm 9.7 \mathrm{~cm}$ (166-188) were submitted to a fitness test which was performed in the soccer field.

The players were assessed by a video-camera, in the field, during the match-play with lactate evaluation. The alactic metabolic pathway was evaluated by means of five $30 \mathrm{~m}$ sprints, in maximum velocity and one minute of passive pause between each sprint. The lactic acid concentrations were assessed at one, three and five minutes after the five sprints had been finished. To detect anaerobic threshold (AT), three $1,200 \mathrm{~m}$ races were performed at an intensity of 80,85 and 90\% from de maximum speed to that distance, with 5 minutes intervals between each running.

The soccer players underwent individual film recording by a video-camera, during the match-play and the lactic acid concentrations were assessed before, during the interval and at the end of the match, to analyze the metabolic and energetics expenditure. The following results were verified: 1) the AT at running, relationed to 4 mmol. $L^{-1}$ lactic acid concentration, was found at $268 \pm 28 \mathrm{~m}^{\mathrm{min}} \mathrm{m}^{-1}$ or $16.1 \pm 1.6$ $\left.\mathrm{km} . \mathrm{h}^{-1} ; 2\right)$ the average speed and the maximum lactate concentration at $30 \mathrm{~m}$ sprint were $6.9 \pm 0.2$ to $4.5 \pm 1.0$ mmol. $L^{-1}$, respectively; 3) the total performed distance was $10,392 \pm 849 \mathrm{~m}, 5,446 \pm 550 \mathrm{~m}$ was found in the first half of the match and 4,945 $\pm 366 m$ was found in the second half of the match; 4) the average values found in assessing blood lactate concentrations were $1.58 \pm 0.37 ; 4.5 \pm 0.42$ and 3.46 \pm 1.54 mmol. $L^{-1}$; before, during the first to the second interval and at the end of the match, respectively; 5) the total average distance approached at the end of the matches by midfield players $(10,910 \pm 121 \mathrm{~m})$ was slightly greater than the one reached by the forward players $(10,377 \pm 224 \mathrm{~m})$ and defenders $(9,889 \pm 102 \mathrm{~m})$, nevertheless, it was not significant. There was a negative correlation $(r=-0.84 ; p<$ $0.05)$ among the AT $\left(268 \pm 28 \mathrm{~m} . \mathrm{min}^{-1}\right.$ or $\left.16.1 \pm 1.6 \mathrm{~km} . \mathrm{h}^{-1}\right)$ and blood lactate concentration $\left(4.5 \pm 0.42 \mathrm{mmol} . \mathrm{L}^{-1}\right)$ in the first half of the match-play.

Thus, the results suggest that the aerobic capacity is an important determinant, which leads the soccer players to cover the long distance runnings in the matches, and be able to recover earlier from the high intensity effort with a lower development of blood lactic acid concentrations in the whole match-play.

Key words: Professional soccer players. Lactate. Anaerobic threshold. Covered distance. Sports medicine.

\section{INTRODUÇÃO}

Estudos arqueológicos permitem afirmar que um jogo de bola praticado com os pés era conhecido no Egito e Babilônia há mais de 30 séculos, cujas descrições de origem variam, segundo alguns autores e estudiosos da matéria.

O futebol com suas características atuais surgiu por volta de 1860 (século XIX). A Federation International of Football Association (FIFA), entidade que governa esse esporte, foi fundada em 1904 e congrega a filiação de mais de 150 países. Portanto, o futebol é um esporte universal ${ }^{1}$.

O futebol é um esporte dinâmico, que requer elevado grau de habilidade técnica, alto nível de aptidão atlética e apurada disciplina tática. A essência desse esporte é a mesma em todo o mundo. Entretanto, as diferenças nas necessidades físicas da época presente, comparadas ao passado, são evidentes. Apesar de ter ultrapassado a barreira dos 130 anos, há ainda muita controvérsia sobre os requerimentos fisiológicos, nível ótimo de treinamento e os métodos de condicionamento físico utilizados no futebol.

Segundo Tumilty ${ }^{1}$, possíveis razões incluem: a) ênfase em demasia dada à habilidade técnica do futebolista; b) negligência no desenvolvimento das qualidades físicas importantes para o jogador; c) dificuldade de aceitação da introdução de metodologias científicas e d) conservadorismo exagerado de alguns preparadores físicos e técnicos, que treinam seus futebolistas (igualmente quando eles eram jogadores de futebol) sem critério e/ou fundamentação científica.

Ao enfatizarmos alguns fatores observados em estudos científicos sobre atletas de alto rendimento, pode-se verificar, cada vez mais, a importância da ciência aplicada ao desenvolvimento e/ou aperfeiçoamento da performance atlética. Para que haja crescimento e evolução no futebol como em outros esportes, é necessário que os profissionais dirijam sua atenção para estudos científicos, pois, somente baseando-se em critérios muito bem fundamentados poderemos utilizar estratégias mais seguras e dar melhores condições de apoio ao atleta.

Sendo o futebol um esporte no qual a vitória é um aspecto muito importante, temos que nos assegurar de que a percentagem de acertos seja a maior possível. Se há um planejamento bem delineado e alicerçado em conceitos bem definidos, então, podemos cobrar resultados.

Nosso estudo procurou trazer através de duas metodologias (videofilmagem e dosagens de lactato sanguíneo) informações que possam qualificar melhor as necessidades de treinamento e o aperfeiçoamento do desempenho físico de jogadores de futebol, fora e dentro de campo.

\section{OBJETIVO}

O propósito deste estudo foi verificar o perfil dos jogadores de futebol, analisado por meio de teste de aptidão física, em campo, relacionado à capacidade funcional, desempe- 
nho energético e solicitação metabólica pela verificação da concentração de lactato sanguíneo e análise cinematográfica, monitorados em situação real de jogo.

\section{MATERIAL E MÉTODOS}

Foram estudados seis jogadores de futebol profissional, todos do sexo masculino, com média de idade de 20,8 $\pm 2,6$ anos (17-25), peso 70,4 $\pm 7,5 \mathrm{~kg}(63-81,3)$ e estatura de 173,3 $\pm 9,7 \mathrm{~cm}$ (166-188). Previamente ao início do estudo, os jogadores foram submetidos a três fases de avaliação, por meio de protocolo elaborado pelo o laboratório de biodinâmica da Universidade Estadual Paulista (Unesp). Os testes foram divididos em três etapas: na primeira, com o objetivo de medir a eficiência do metabolismo anaeróbio alático, os jogadores realizaram cinco corridas na distância de $30 \mathrm{~m}$, em velocidade máxima e com pausa passiva de um minuto entre cada corrida. Foram coletadas amostras de sangue para análise da

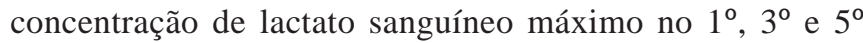
minuto após o término das cinco corridas; e na segunda, com a finalidade de verificar o limiar anaeróbio, os futebolistas realizaram três corridas na distância de $1.200 \mathrm{~m}$, com velocidades preestabelecidas nas intensidades de 80,85 e $90 \%$ da velocidade máxima para esse percurso, com intervalo de recuperação passiva de, no mínimo, 15 minutos entre cada corrida. O teste foi realizado em pista de atletismo de $400 \mathrm{~m}$, na qual o ritmo foi controlado por meio de um apito a cada $100 \mathrm{~m}$. Foram coletadas amostras de sangue para análise de

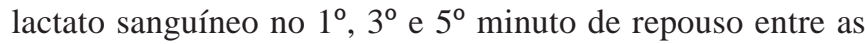
corridas. O critério utilizado para a determinação do limiar anaeróbio foi a interpolação linear da velocidade média de corrida correspondente à concentração de lactato sanguíneo de $4 \mathrm{mmol} . \mathrm{L}^{-1}{ }^{2}$. Em todos os testes, o sangue arterial do lóbulo de orelha foi coletado sem hiperemia, em tubo capilar heparinizado e calibrado para $25 \mu \mathrm{l}$ e imediatamente diluído em $50 \mu 1$ de solução de fluoreto de sódio (1\%) mantido em gelo. As amostras foram colhidas em tubos de plástico tipo Eppendorf e mantidas em gelo para posterior análise por meio de método eletroquímico pelo aparelho da marca Yellow Spring, Inc., USA, modelo YSL 2300 STAT. A terceira etapa deste estudo objetivou analisar o desempenho energético e metabólico dos futebolistas em situação real de jogo. Cada jogador foi observado por meio de filmagem durante toda a sua atuação na partida através de câmera de vídeo. As filmagens foram padronizadas de maneira que permitissem identificar facilmente a localização do futebolista. Portanto, foram padronizados a filmagem, o jogador e as linhas (medidas) do campo para serem utilizadas como referências de localização. Esse procedimento foi utilizado para permitir a verificação do deslocamento total ao final do jogo (distância percorrida). As fitas dos jogos foram reproduzidas na condição de slow motion em monitor de TV de 20 polegadas e videocassete de 4 cabeças com velocidade de 1:6. Todas as variáveis foram obtidas com auxílio de um programa de computador desenvolvido pela Universidade Estadual Paulista (Unesp) Campus de Rio Claro - SP. A posição do jogador foi plotada na tela do computador a cada segundo, em representação do campo de futebol numa escala de 5 metros em dimensões reais $(99 \mathrm{~m} \times 60 \mathrm{~m})$. A cada minuto (60 pontos) passava-se para a tela seguinte e assim sucessivamente até o último minuto da partida analisada. A posição dos jogadores foi transformada em coordenadas cartesianas. As distâncias foram calculadas através do teorema de Pitágoras para triângulos retângulos. A solicitação metabólica em situação real de jogo foi medida por meio de lactato sanguíneo, antes do jogo após aquecimento, no intervalo do primeiro para o segundo tempo e ao final da partida.

Análise estatística: Todos os dados estudados foram submetidos ao teste de correlação linear de Pearson, coeficiente de Spearmann e ao teste $t$ de Student para determinação de correlação e diferença significante entre todas as variáveis analisadas $^{3}$.

\section{RESULTADOS}

Testes de campo: A velocidade média atingida na distância de $30 \mathrm{~m}$ foi de $6,9 \pm 0,2 \mathrm{~m} \cdot \mathrm{s}^{-1}$ ou $24,8 \mathrm{~km} \cdot \mathrm{h}^{-1}$, com variação mínima e máxima de 6,6 a $7,1 \mathrm{~m} \cdot \mathrm{s}^{-1}\left(23,7-25,5 \mathrm{~km} \cdot \mathrm{h}^{-1}\right)$, respectivamente (tabela 3 ). A concentração média de lactato sanguíneo ao final de cinco corridas de $30 \mathrm{~m}$ foi de $4,5 \pm$ $1,0 \mathrm{mmol} . \mathrm{L}^{-1}$, com variação mínima e máxima de 3,4 a 6,3 mmol. $\mathrm{L}^{-1}$, respectivamente (tabela 3 ). O limiar anaeróbio foi considerado no ponto em que a concentração fixa de lactato sanguíneo alcançou $4 \mathrm{mmol} . \mathrm{L}^{-1}$ e ocorreu numa velocidade média de corrida de $268 \pm 28 \mathrm{~m} \cdot \mathrm{min}^{-1}$ ou $16,1 \pm 1,6 \mathrm{~km} \cdot \mathrm{h}^{-1}$,

\begin{tabular}{|c|c|c|c|}
\hline \multicolumn{4}{|c|}{$\begin{array}{c}\text { TABELA } 1 \\
\text { Distância total percorida em metros (m) ao final } \\
\text { do primeiro e segundo tempo de uma partida } \\
\text { de futebol verificada neste estudo }\end{array}$} \\
\hline \multirow{2}{*}{$\frac{\text { Futebolista }}{\text { No }}$} & \multicolumn{3}{|c|}{ Distância percorrida (m) } \\
\hline & $\begin{array}{l}\text { Primeiro } \\
\text { tempo }\end{array}$ & $\begin{array}{l}\text { Segundo } \\
\text { tempo }\end{array}$ & Total \\
\hline 1 & 5.380 & 4.673 & 10.053 \\
\hline 2 & 6.232 & 5.535 & 11.767 \\
\hline 3 & 4.632 & 4.534 & 9.166 \\
\hline 4 & 5.121 & 5.098 & 10.219 \\
\hline 5 & 5.526 & 5.086 & 10.612 \\
\hline 6 & 5.788 & 4.748 & 10.536 \\
\hline$N=6$ & $\begin{array}{c}5.446 * \\
\pm 550 \\
(4.632-6.232)\end{array}$ & $\begin{array}{c}4.945 \\
\pm 366 \\
(4.534-5.535)\end{array}$ & $\begin{array}{c}10.392 \\
\pm 849 \\
(9.166-11.767)\end{array}$ \\
\hline
\end{tabular}

Teste $t$ pareado 1 o vs. 2 o tempo de jogo; $* p<0,05$.

Os resultados representam a média, o desvio-padrão e as variações mínima e máxima. 
com variação mínima e máxima de 237 a 298 m.min-1 (14,2$17,9 \mathrm{~km} . \mathrm{h}^{-1}$ ), respectivamente (tabela 3 ).

Distância percorrida na partida: A distância média alcançada pelos jogadores ao final do primeiro e segundo tempo da partida foi significantemente diferente $(\mathrm{p}<0,05) 5.446$ $\pm 550 \mathrm{~m}$ e $4.945 \pm 336 \mathrm{~m}$, respectivamente (tabela 1 ). A variação mínima e máxima do primeiro tempo foi de 4.632 a $6.232 \mathrm{~m}$ e a do segundo tempo, de 4.534 a $5.535 \mathrm{~m}$, respectivamente (tabela 1 ). Ao final do jogo, a distância média total ( 1 으 e 2 o tempo) foi de $10.392 \pm 849 \mathrm{~m}$, com variação mínima e máxima de 9.166 a $11.767 \mathrm{~m}$, respectivamente (tabela 1 ).

Concentração de lactato sanguíneo durante a partida: A concentração média de lactato sanguíneo verificada no iní-

\begin{tabular}{|c|c|c|c|}
\hline \multicolumn{4}{|c|}{$\begin{array}{c}\text { TABELA } 2 \\
\text { Concentração de lactato sanguíneo no início, intervalo } \\
\text { e final de partida verificada em seis jogadores de } \\
\text { futebol profissional analisados neste estudo }\end{array}$} \\
\hline \multirow{2}{*}{$\frac{\text { Futebolista }}{\text { № }}$} & \multicolumn{3}{|c|}{ Lactato (mmol..-1) } \\
\hline & Início & Intervalo & Final \\
\hline 1 & 1,4 & 4,7 & 3,0 \\
\hline 2 & 1,4 & 3,9 & 2,7 \\
\hline 3 & 1,3 & 4,8 & 2,6 \\
\hline 4 & 1,4 & 4,5 & 6,6 \\
\hline 5 & 2,3 & 4,1 & 3,0 \\
\hline 6 & 1,7 & 5,0 & 2,9 \\
\hline $\mathrm{N}=6$ & $\begin{array}{l}1,58 \\
\pm 0,37 \\
(1,3-2,3)\end{array}$ & $\begin{array}{l}4,50 \\
\pm 0,42 \\
(3,9-5,0)\end{array}$ & $\begin{array}{l}3,46 \\
\pm 1,54 \\
(2,6-6,6)\end{array}$ \\
\hline
\end{tabular}

Os resultados representam a média, o desvio-padrão e as variações mínima e máxima.

TABELA 3

Resultados dos testes de campo na distância de $30 \mathrm{~m}$ (velocidade e lactato sanguíneo) e limiar anaeróbio em velocidade de corrida a concentração fixa de lactato a $4 \mathrm{mmol} . \mathrm{L}^{-1}$ nesse grupo de futebolistas profissionais avaliados

\begin{tabular}{|c|c|c|c|c|c|}
\hline \multirow{2}{*}{$\begin{array}{c}\text { Futebolista } \\
\text { № }\end{array}$} & \multicolumn{2}{|c|}{$\begin{array}{l}\text { Velocidade } \\
\left(\mathbf{m} \cdot \mathbf{s}^{-1} \mathbf{k m} \cdot \mathbf{h}^{-1}\right)\end{array}$} & \multirow{3}{*}{$\begin{array}{c}\begin{array}{c}\text { Lactato } \\
\text { (mmol.L-1) }\end{array} \\
\mathbf{3 0 m} \\
3,6\end{array}$} & \multirow{2}{*}{\multicolumn{2}{|c|}{$\begin{array}{c}\begin{array}{c}\text { Limiar anaeróbio } \\
\left(\mathrm{m} \cdot \mathrm{min}^{-1} \mathbf{~ k m} \cdot \mathrm{h}^{-1}\right)\end{array} \\
4 \mathrm{mmol} \cdot \mathrm{L}^{-1}\end{array}$}} \\
\hline & & Om & & & \\
\hline 1 & 7,1 & 25,5 & & 238 & 14,3 \\
\hline 2 & 6,8 & 24,5 & 4,6 & 298 & 17,9 \\
\hline 3 & 6,8 & 24,5 & 3,4 & 259 & 15,5 \\
\hline 4 & 7,1 & 25,5 & 4,8 & 298 & 17,9 \\
\hline 5 & 7,1 & 25,5 & 4,6 & 281 & 16,9 \\
\hline 6 & 6,6 & 23,7 & 6,3 & 237 & 14,2 \\
\hline$N=6$ & $\begin{array}{c}6,9 \\
\pm 0,2 \\
(6,6-7,1)\end{array}$ & $\begin{array}{c}24,8 \\
\pm 0,7 \\
(23,7-25,5)\end{array}$ & $\begin{array}{c}4,5 \\
\pm 1,0 \\
(3,4-6,3)\end{array}$ & $\begin{array}{c}268 \\
\pm 28 \\
(237-298)\end{array}$ & $\begin{array}{c}16,1 \\
\pm 1,6 \\
(14,2-17,9)\end{array}$ \\
\hline
\end{tabular}

Os resultados representam a média, o desvio-padrão e as variações mínima e máxima. cio, logo após a fase de aquecimento dos futebolistas, foi de $1,58 \pm 0,37 \mathrm{mmol} . \mathrm{L}^{-1}$, com variação de 1,3 a $2,3 \mathrm{mmol} \cdot \mathrm{L}^{-1}$, respectivamente (tabela 2). A segunda medida foi realizada após o término do primeiro tempo da partida, fase denominada de intervalo, e o lactato médio foi de 4,50 $\pm 0,42 \mathrm{mmol}$. $\mathrm{L}^{-1}$, com variação de 3,9 a $5,0 \mathrm{mmol} . \mathrm{L}^{-1}$, respectivamente (tabela 2). A última medida foi realizada logo após o final do jogo e a concentração média foi de $3,46 \pm 1,54 \mathrm{mmol} . \mathrm{L}^{-1}$, com variação de 2,6 a $6,6 \mathrm{mmol} . \mathrm{L}^{-1}$, respectivamente (tabela 2).

\section{DISCUSSÃO}

A metodologia de registro do desempenho físico por análise cinematográfica, utilizando-se vídeo e computador, é um meio eficaz que permite através das informações desenvolver o programa de treinamento físico dos futebolistas com maior precisão, pois é possível quantificar, em volume e intensidade, os vários ritmos que caracterizam o rendimento do jogador de futebol durante uma partida em tempo real.

Os testes de aptidão física, realizados em campo e laboratório de fisiologia, são de grande importância, pois permitem o controle evolutivo e corrigem possíveis deficiências ao longo do treinamento dos jogadores. Portanto, é um meio adequado de controlar melhor a evolução das valências físicas necessárias aos jogadores, com exercícios de melhor qualidade, preservando sobretudo a integridade física do futebolista. Em nosso estudo, podemos verificar índices de aptidão física importantes para jogar futebol com elevado rendimento atlético. O limiar anaeróbio (LA) determinado quando a concentração de lactato sanguíneo atingiu $4 \mathrm{mmol} . \mathrm{L}^{-1}$, em campo, foi superior à média encontrada em jogadores de basquete, futebol de campo e de futsal, respectivamente ${ }^{4-6}$.

O LA é uma zona metabólica a partir da qual ocorre um desequilíbrio entre a produção e a eliminação do lactato. A sua determinação, seja por troca ventilatória (ergoespirometria) ou por dosagem de lactato, tem implicações práticas importantes na prescrição e avaliação dos efeitos do treinamento físico para atletas em diversas modalidades esportivas. No futebol, a detecção do LA tem sido verificada rotineiramente pelos dois métodos. Ambos são válidos e apresentam elevado grau de correlação quando comparados ${ }^{7-10}$.

Em nosso estudo, a velocidade média de $268 \mathrm{~m} \cdot \mathrm{min}^{-1}$ ou $16,1 \mathrm{~km} \cdot \mathrm{h}^{-1}$, verificada no LA, foi superior aos achados de Bangsbo ${ }^{10}$, que avaliou 60 jogadores dinamarqueses considerados de elite, verificou a velocidade média de $14,5 \mathrm{~km} . \mathrm{h}^{-1}$ a uma concentração fixa de lactato sanguíneo a $3,0 \mathrm{mmol} . \mathrm{L}^{-1}$, em teste realizado na esteira, na posição horizontal (sem inclinação). Entretanto, quando dividiu os jogadores por posição, encon- 
trou resultados semelhantes aos nossos, ou sejam, 16,1 vs. $15,9 \mathrm{~km} \cdot \mathrm{h}^{-1}$. Em outro estudo, realizado por Green ${ }^{11}$, foi confirmado que jogadores mais qualificados atingiram valores de LA, em velocidade de corrida, mais altos, sendo este um índice de grande importância para qualificar o grau de aptidão física do futebolista. $\mathrm{Na}$ opinião de Bunc et al. ${ }^{12}$, para jogadores de futebol atingirem um bom condicionamento físico para a prática desse esporte, em nível competitivo, necessitam ter índices de LA, em velocidade de corrida, superiores a $14 \mathrm{~km} \cdot \mathrm{h}^{-1}$. Além disso, com relação ao rendimento físico, quanto maior a percentagem do $\mathrm{VO}_{2} \mathrm{em}$ relação ao $\mathrm{VO}_{2} \max$., no LA, maior a probabilidade de sucesso físico durante a partida, pois essas duas condições são alguns dos fatores considerados preditores de uma boa capacidade do organismo para tolerar a longa duração do jogo, com maior eficiência de movimento, sem que o jogador canse rapidamente.

Outra avaliação importante utilizada neste estudo foi o teste na distância de $30 \mathrm{~m}$. A velocidade média nas corridas de $30 \mathrm{~m}\left(6,9 \pm 0,2 \mathrm{~m} \cdot \mathrm{s}^{-1}\right.$ ou $\left.24,8 \pm 0,7 \mathrm{~km} \cdot \mathrm{h}^{-1}\right)$ foi menor que a encontrada nos estudos de Kokubun \& Daniel ${ }^{4}$; Chelles ${ }^{5}$ e Molina $^{6}$, realizados em jogadores de basquete, futebol de campo e futsal, respectivamente. No entanto, foi superior à média encontrada nesse mesmo tipo de teste realizado em jogadores da seleção nacional da Jamaica $6,6 \pm 0,16 \mathrm{~m} \cdot \mathrm{s}^{-1}$ (dados não publicados).

A concentração de lactato ao final das corridas de $30 \mathrm{~m}$ $\left(4,5 \pm 1,0 \mathrm{mmol} . \mathrm{L}^{-1}\right)$ foi inferior, quando comparada à média dos jogadores de basquete, futebol de campo e futsal, respectivamente ${ }^{4-6}$.

De acordo com Fohrenbach et al..$^{13}$, o desempenho nas corridas de $30 \mathrm{~m}$ com um minuto de pausa, em jogadores de futebol com melhor condicionamento físico aeróbio, apresenta concentração de lactato menor e mais velocidade. Nas corridas de $30 \mathrm{~m}$, o tempo de esforço é pequeno para que ocorra eficientemente a ativação da via glicolítica anaeróbia. Além disso, o tempo de recuperação de um minuto é longo, havendo portanto a reposição das reservas de fosfagênios (ATP-CP). É importante ressaltar que alguns autores $^{14-17}$ verificaram que níveis elevados de potência aeróbia exercem importante papel na recuperação mais rápida da energia proveniente do sistema fosfagênio (ATP-CP), responsável por considerável fornecimento de energia durante períodos de alta intensidade, como também remoção mais eficiente do lactato nos momentos de repouso ativo e/ou diminuição da intensidade do exercício. Porém, após várias corridas nessa distância, em alta intensidade, as reservas de fosfagênios (ATP-CP) musculares diminuem significativamente, induzindo à participação da via glicolítica anaeróbia e, conseqüentemente, aumento da produção de lactato. Contudo, jogadores que apresentam potência aeróbia bem desenvolvida produzem menos lactato em qualquer intensidade de exercício ${ }^{18}$. Essa afirmação confirma os resultados mostrados neste estudo, pois índices de LA, em velocidade de corrida, foram relativamente altos para a categoria e, ao contrário, os valores de concentrações de lactato sanguíneo foram relativamente baixos nas corridas de $30 \mathrm{~m}$ quando comparados a outros estudos. A justificativa para essa baixa concentração de lactato sanguíneo talvez tenha sido o grande volume de treinamento aeróbio realizado por esses jogadores, inibindo consequientemente a via metabólica lática (ver tabela 3 ).

Um dos meios utilizados para estimar a solicitação energética requerida pela intensidade de esforço durante uma partida de futebol é a verificação da distância total percorrida ao final de um jogo. Portanto, alguns autores preocuparam-se em investigar o quanto um jogador de futebol se desloca em campo durante uma partida (ver quadro 1). Em adição, alguns estudos direcionaram seus objetivos para descobrir o tempo gasto, o número de deslocamentos e a percentagem desses movimentos em condições de baixa e alta intensidade durante a partida.

A história científica e esportiva registrou, por meio de Winterbottom (1959), que foi citado por Bangsbo ${ }^{10}$, a primeira tentativa de verificar qual era a distância percorrida por jogadores de futebol profissional durante a partida. Seus estudos verificaram uma distância média de $3.361 \mathrm{~m}$, enquanto Wade ${ }^{19}$ encontrou valores que variaram de 1.600 a $5.486 \mathrm{~m}$.
QUADRO 1

Distância percorrida e métodos utilizados por vários autores para a verificação do desempenho físico em jogadores de futebol

\begin{tabular}{|c|c|c|c|}
\hline $\begin{array}{c}\text { Autor } \\
\text { do estudo }\end{array}$ & $\begin{array}{c}\text { № de futebolistas } \\
\text { analisados }\end{array}$ & $\begin{array}{c}\text { Distância } \\
\text { percorrida }(\mathrm{m})\end{array}$ & $\begin{array}{l}\text { Método } \\
\text { utilizado }\end{array}$ \\
\hline 1) Knowles \& Brooke 58 & 40 & 4.834 & Anotação manual \\
\hline 2) Smaros 52 & 7 & 7.100 & Câmeras de TV (2) \\
\hline 3) Reilly \& Thomas 28 & 40 & 8.680 & Videoteipe \\
\hline 4) Ekblom 20 & 10 & 9.800 & Anotação manual \\
\hline 5) Ohashi et al.29 & 2 & 9.845 & Trigonometria (2 câmeras) \\
\hline 6) Van Gool et al.23 & 7 & 10.245 & Filmagem \\
\hline 7) Bangsbo et al. ${ }^{30}$ & 14 & 10.800 & Vídeo (24 câmeras) \\
\hline 8) Saltin31 & 9 & 10.900 & Filmagem \\
\hline 9) Zelenka et al.56 & 1 & 11.500 & Não revelado \\
\hline 10) Withers et al. ${ }^{22}$ & 20 & 11.527 & Videoteipe \\
\hline 11) Ohashi et al. 57 & 50 & 11.529 & Trigonometria \\
\hline
\end{tabular}


Com o decorrer dos anos, o aparecimento de técnicas de análise mais precisas, a evolução tática e, principalmente, física dos futebolistas, essas distâncias médias aumentaram.

Agnevik (1970), citado por Ekblom ${ }^{20}$, após investigar jogadores da primeira e segunda divisões da Suécia, encontrou distância média de $10.200 \mathrm{~m}$. Resultados semelhantes foram encontrados por Whitehead ${ }^{21}$, em futebolistas da primeira e segunda divisões da Inglaterra, com valores de 13.500 e $11.200 \mathrm{~m}$, respectivamente.

Ao analisar jogadores profissionais da Austrália, Withers ${ }^{22}$ encontrou média de $11.500 \mathrm{~m}$ para distância percorrida durante a partida, sendo $18,8 \%$ realizados em alta intensidade. O mesmo autor e colegas, em 1982, verificaram que os jogadores que atuaram no meio-campo percorreram uma distância maior em relação aos defensores e atacantes, ou seja, $12.194 \mathrm{~m}, 10.169 \mathrm{~m}$ e $11.766 \mathrm{~m}$, respectivamente.

No presente estudo, a distância total média atingida pelos jogadores durante jogo foi de $10.392 \pm 849 \mathrm{~m}$. Esse resultado está próximo dos encontrados por vários autores ${ }^{20,22-24} \mathrm{em}$ jogadores de futebol profissional de outros países e muito superior aos encontrados em jogadores de basquetebol e fut$\mathrm{sal}^{4-6}$.

O estudo de Mayhew \& Wenger ${ }^{25}$ analisou dois jogadores que atuavam na posição de meio-campo em uma equipe norte-americana. Os dois futebolistas foram filmados por uma câmera, cada um, durante 42 minutos. As observações foram realizadas e passadas diretamente do vídeo para o computador. A técnica utilizada possibilitou estimar: a) o tempo gasto em cada atividade; b) o número de intervenções físicas da partida; e a c) percentagem total do tempo gasto nos vários tipos de movimentos. Eles classificaram a movimentação dos futebolistas da seguinte maneira: 1) parado (sem movimento); 2) andando (avanços, passos laterais e para trás); 3) trotando (corrida lenta em que o jogador não tinha objetivo específico); 4) correndo (combinação entre passo largo e corrida com objetivo e grande esforço) e 5) utilitários (combinação de corridas para trás, combinada lateral e salto).

As observações do trabalho de Yamanaka et al. ${ }^{26}$ foram feitas utilizando-se 49 jogadores, divididos em três grupos (A, B e C) e filmados durante toda a partida. Os movimentos foram classificados como: 1) parado; 2) andando; 3) trotando (em condição de corrida a baixa velocidade); 4) correndo (corrida com objetivo definido e intermediária entre corrida apressada e rápida); e por fim 5) corrida em alta velocidade.

Mais recentemente, D'Ottavio \& Tranquilli ${ }^{27}$ analisaram um futebolista, meio-campista da seleção italiana sub-21. Nesse estudo, foram utilizadas duas câmeras que registraram as imagens. Através de um software calculou-se a distância percorrida em quilômetros e os vários tipos de deslocamentos. Eles classificaram a movimentação desse atleta da seguinte maneira: 1) parado; 2) andando a $5 \mathrm{~km} \cdot \mathrm{h}^{-1} ; 3$ ) corrida lenta a velocidade de $11 \mathrm{~km} \cdot \mathrm{h}^{-1}$; 4) corrida média a $15 \mathrm{~km} \cdot \mathrm{h}^{-1}$; 5) corrida a passo largo a $18 \mathrm{~km} \cdot \mathrm{h}^{-1} ; 6$ ) corrida rá- pida a $21 \mathrm{~km} \cdot \mathrm{h}^{-1}$; 7) sprint a $24 \mathrm{~km} \cdot \mathrm{h}^{-1}$; 8) sprint máximo a 27 $\mathrm{km} . \mathrm{h}^{-1}$; corrida em retrocesso de $3 \mathrm{a} 21 \mathrm{~km} \cdot \mathrm{h}^{-1}$ e lateral de 0 a $21 \mathrm{~km} . \mathrm{h}^{-1}$. Ao final da partida, os cálculos mostraram que esse futebolista percorreu a distância de $12.060 \mathrm{~m}$.

A média da distância percorrida pelos futebolistas no presente trabalho, durante o primeiro e o segundo tempo, foi de $5.446 \pm 550 \mathrm{~m}$ e $4.945 \pm 366 \mathrm{~m}$, respectivamente. Encontrouse diferença significativa $(\mathrm{p}<0,05)$ nas distâncias percorridas entre os dois períodos (ver tabela 1). Essa diferença está de acordo com os resultados obtidos por Reilly \& Thomas ${ }^{28}$, Ohashi et al..$^{29}$; Van Gool et al..$^{23}$ e Bangsbo et al. ${ }^{10}$. Esses resultados sugerem que há um declínio do rendimento físico ao final da partida. Isso pode ser provocado por uma diminuição na concentração de glicogênio muscular, que pode ocorrer já no primeiro tempo do jogo, como observou Sal$\operatorname{tin}^{31}$.

Quando analisamos o desempenho dos jogadores, de acordo com a posição (defensor, meio-campista e atacante) adotada na partida, encontramos diferença na distância percorrida entre eles. Os futebolistas meio-campistas apresentaram resultados maiores que os dos atacantes e defensores (10.910 $\pm 121 \mathrm{~m} ; 10.377 \pm 224 \mathrm{~m}$ e $9.889 \pm 102 \mathrm{~m}$, respectivamente). Esses valores são semelhantes aos encontrados por Ekblom ${ }^{20}$ e Van Gool et al. ${ }^{23} \mathrm{em}$ jogadores profissionais da Suécia e universitários da Bélgica, respectivamente. Portanto, a variação de movimentos e a distância total percorrida pelo jogador ao final do jogo é de grande utilidade para orientar a carga de treinamento físico semanal realizada por esses atletas.

A metodologia de videofilmagem das atividades realizadas numa partida de futebol tem a vantagem de estabelecer o percurso total e/ou partes deste, facilitando a avaliação e trazendo informações que podem ser canalizadas para melhor qualificar o treinamento diário do futebolista.

Outro meio utilizado para se verificar a resposta metabólica e o conseqüente dispêndio energético de futebolistas durante uma partida de futebol tem sido a dosagem de lactato sanguíneo, que é produzido durante exercício de alta intensidade. Entretanto, a questão é saber quanto de energia é derivada da glicólise e sua conseqüente formação de lactato para o rendimento do futebolista. A concentração desse metabólito no sangue representa o balanço entre a produção e a remoção. Quando ele é medido, estamos tentando avaliar a sua concentração como indicador de produção.

Entretanto, vários fatores devem ser considerados. O lactato é metabolizado dentro dos músculos ativos após exercício de alta intensidade ${ }^{32,33}$. Contudo, nem todo o lactato liberado pelos músculos ativos vai para a corrente sanguínea, pois determinada concentração está sendo distribuída a taxas elevadas para outros tecidos como o coração, fígado, rins e músculos inativos ${ }^{32}$.

Outro problema está relacionado à duração do exercício intenso, que pode ser curto, para provocar considerável au- 
mento do lactato. Boobis ${ }^{34}$ observou que a concentração de lactato dentro do músculo aumentou aproximadamente $7 \mathrm{mmol} . \mathrm{kg}^{-1}$.w.w $\left( \pm 10 \mathrm{mmol} . \mathrm{L}^{-1}\right)$ durante um sprint de 6 segundos, enquanto a concentração verificada no sangue foi somente de $1,8 \mathrm{mmol} . \mathrm{L}^{-1}$ e no período de recuperação não passou de $5 \mathrm{mmol} . \mathrm{L}^{-1}$.

Alguns autores acreditam ${ }^{35-39}$ que a baixa concentração de lactato no sangue é, provavelmente, devida a uma limitada liberação e a um grande espaço de difusão para o seu deslocamento, já que, comparativamente, a sua concentração é bem maior no músculo que no sangue, tanto em exercício submáximo como no máximo ${ }^{40-45}$.

$\mathrm{Na}$ maioria dos estudos em que os pesquisadores dosaram a concentração de lactato sanguíneo em situação de jogo, encontrou-se um valor menor no segundo, comparado ao primeiro tempo da partida ${ }^{46,47}$. Resultados semelhantes foram encontrados em nosso estudo. As concentrações verificadas para os dois tempos foram de 4,50 $\pm 0,42$ vs. 3,46 $\pm 1,54$ mmol. $\mathrm{L}^{-1}$, respectivamente. Entretanto, a justificativa para essa diminuição, na segunda metade do jogo, está relacionada, segundo Bangsbo ${ }^{10}$, a menor distância atingida $(4,7 v s$. $5,4 \mathrm{~km})$ e à menor realização de corridas em alta intensidade (0,83 vs. $1,24 \mathrm{~km})$, no segundo tempo da partida. Uma resposta cardiovascular mais baixa também parece acontecer em decorrência disso, como, por exemplo, uma diminuição de 10 batimentos na frequiência cardíaca. Além disso, a baixa concentração de glicogênio muscular é um fator potencial para a diminuição do desempenho físico dos futebolis$\operatorname{tas}^{48}$.

O glicogênio muscular exerce importante papel em exercícios ou atividades que solicitem elevada percentagem do consumo máximo de oxigênio ( $\mathrm{VO}_{2} \max$.). Sendo assim, as reservas de glicogênio começam a diminuir significativamente. A sua utilização varia de acordo com tipo de modalidade esportiva, estado de treinamento, proporção utilizada de fibras de contração lenta e rápida e suas reservas iniciais ${ }^{48,49}$.

Reilly $^{50}$ afirma que ocorre um declínio nos níveis de glicogênio muscular no $1 \stackrel{0}{\text { }}$ tempo da partida, predominantemente nas fibras de contração lenta ${ }^{51}$, sugerindo que o metabolismo aeróbio pode atuar por longo período utilizando glicogênio muscular para produzir energia. Portanto, desde que a solicitação pelo metabolismo aeróbio no futebol é considerada elevada, é de grande importância aumentar as reservas de glicogênio antes da partida ${ }^{20-48}$.

Jacobs et al., citados por Ekblom ${ }^{20}$, observaram após uma partida de futebol que a reposição do glicogênio era muito baixa, mesmo após 48 horas. Os autores atribuíram esses valores à baixa concentração de carboidratos na dieta dos futebolistas.

Apesar da dificuldade para medir a concentração do lactato real no músculo e no sangue, tem sido observada uma grande variação deste último, em futebolistas durante o jogo. Entretanto, valores acima de $10 \mathrm{mmol} . \mathrm{L}^{-1}$ de lactato sanguí- neo têm sido verificados na literatura especializada. Agnevik, citado por Ekblom ${ }^{20}$, verificou em jogadores da primeira divisão da Suécia, ao final do jogo, concentração entre 10 e $15,5 \mathrm{mmol} . \mathrm{L}^{-1}$; Ekblom ${ }^{20}$, encontrou ao final do primeiro e segundo tempo de partidas de futebol, em quatro divisões da liga sueca, as seguintes concentrações de lactato sanguíneo médias e variações: primeira $(9,5[6,9-14,3])$ vs. $(7,2[4,5-$ $\left.\left.10,8 \mathrm{mmol.L} \mathrm{L}^{-1}\right]\right)$; segunda $(8,0[5,1-11,5])$ vs. $(6,6[3,1-$ $\left.\left.11,0 \mathrm{mmol} . \mathrm{L}^{-1}\right]\right)$; terceira $(5,5[3,0-12,6])$ vs. $(4,2[3,2-$ $\left.\left.8,0 \mathrm{mmol} . \mathrm{L}^{-1}\right]\right)$ e quarta divisão $(4,0[1,9-6,3])$ vs. $(3,9[1,0-$ $\left.\left.8,5 \mathrm{mmol} . \mathrm{L}^{-1}\right]\right)$. Portanto, o desempenho físico está na dependência da freqüência de envolvimento do futebolista em exercícios de alta intensidade, com duração suficiente para solicitar a via metabólica lática e de seu adequado estoque de glicogênio muscular.

Sendo assim, podemos deduzir que a baixa concentração de lactato sanguíneo verificada em alguns estudos, ao final de uma partida de futebol, poderá estar associada a alguns fatores como: a) elevada capacidade aeróbia, que inibe a via glicolítica lática; b) pouco envolvimento em exercícios de alta intensidade e estímulo insuficiente para ativar a via metabólica lática; c) baixa concentração dos estoques de glicogênio muscular, diminuindo a capacidade do futebolista em exercer esforço intenso; d) níveis sanguíneos aumentados de amônia, hipoxantina e ácido úrico, que são metabólitos considerados preditores de fadiga central e muscular (periférica), conforme estudos de vários autores ${ }^{10,52-55}$.

\section{CONCLUSÃO}

Pelas observações feitas neste estudo e que estão de acordo com vários autores na literatura especializada em futebol, podemos sugerir que: 1) O futebolista parece precisar de uma boa capacidade aeróbia (endurance), já que a distância atingida ao final das partidas pelos jogadores analisados neste estudo foi grande; além disso, recuperam-se mais rapidamente os futebolistas que realizam esforços em alta intensidade; 2) Apesar de alguns estudos relatarem apenas uma modesta solicitação anaeróbia, com exercícios de alta intensidade durante as partidas de futebol, outros têm demonstrado participação alta desse metabolismo, sendo verificada concentração elevada de lactato sanguíneo de até $16 \mathrm{mmol}^{-1}$, ao final de uma partida de futebol. Portanto, o futebol jogado na atualidade parece exigir dos jogadores desse esporte boas condições para tolerar exercícios na presença de lactato sanguíneo; 3) Face à longa duração e intermitência do jogo, é necessário reposição adequada dos depósitos de glicogênio muscular antes, durante e após as partidas, pois a diminuição deste nutriente, comprovadamente, reduz significativamente o rendimento físico do futebolista, principalmente no $2 \underline{0}$ tempo das partidas; e 4) Portanto, para o futebolista resistir à longa duração do jogo, exercendo bom ritmo de movimentação, em condições de força e velocidade, necessita desen- 
volver eficientemente os metabolismos aeróbio, anaeróbio alático e lático, respectivamente.

\section{AGRADECIMENTOS}

Agradecemos à professora Angela Romano, da seção de ergometria do Instituto do Coração (InCor), Unidade Divino Salvador, do Hospital das Clínicas da Faculdade de Medicina da Universidade de São Paulo (HCFMUSP), por sua colaboração.

\section{REFERÊNCIAS}

1. Tumilty D. Physiological characteristics of elite soccer players. Sports Medicine 1993;16:80-96.

2. Heck H. Justification of the $4 \mathrm{mmol} /$ lactate threshold. J Sports Medicine 1985;6:117-30

3. Glantz SA. Primer of biostatistics. $3^{a}$ ed. New York: Mc Graw-Hill, 1992.

4. Kokubun E, Daniel JF. Relações entre a intensidade e duração das atividades em partida de basquetebol com as capacidades aeróbica e anaeróbica: estudo pelo lactato sanguíneo. Rev Paul Educ Fis 1992;6:37-46.

5. Chelles C. Capacidade aeróbica e anaeróbica no futebol: avaliação através do lactato. Rio Claro: Unesp, 1992:37 p, Monografia (Licenciatura em Educação Física e Esportes) Instituto de Biociências, Universidade Estadual Paulista, 1992.

6. Molina R. Futsal: um estudo das capacidades aeróbicas e anaeróbicas de jogadores e das atividades em jogo. Rio Claro: Unesp, 1992:57 p, Monografia (Bacharelado em Educação Física) Instituto de Biociências, Universidade Estadual Paulista, 1992.

7. Reinhard U, Muller PH, Schumulling RM. Determination of anaerobic threshold by ventilation equivalent in normal individuals. Respiration 1979;38:36-42.

8. Anderson GS, Rhodes EC. A review of blood lactate and ventilatory methods of detecting transition thresholds. Sports Med 1989;8:43-55.

9. Christopher ER, Loar CER, Rhodes EC. Relationship between the lactate and ventilatory threshold during prolonged exercise. Sports Med 1993;15:104-15

10. Bangsbo J. Energy demands in competitive soccer. J Sports Sci 1994; 12:5-12.

11. Green S. Anthropometric and physiological characteristics of South Australian soccer players. Aust J Sci Med Sport 1992;24:3 -7.

12. Bunc V, Heller J, Leso J, Sprynarova S, Zdanowicz R. Ventilatory threshold in various groups of higly trained athletes. Int J Sports Med 1987;8: 275-80.

13. Forhenbach R. Testverhfahren und metabolisch orientierte intensitatssteurung in sprinttraining mit submaxiler Belastungsstruktur. Leistungssport 1986;5:15-24.

14. Donovan CM, Brooks GA. Endurance training affects lactate clearance, not lactate production Am J Physiol 1983;244:E83-E92.

15. Donovan CM, Pagliassotti MJ. Endurance training enhances lactate clearance during hyperlactatemia. Am J Physiol 1989;257:E782-E89.

16. Donovan CM, Pagliassotti MJ. Enhance efficiency of lactate removal after endurance training. J Appl Physiol 1990;68:1053-8.

17. Mac Rae HSH, Dennis SC, Bosch AN, Noakes TD. Effects of training in lactate production and removal during progressive exercise in humans. J Appl Physiol 1992;72:1649-56.

18. Jabobs I. Blood lactate implications for training and sports performance. Sports Med 1986;3:10-25.
19. Wade A. The training of young players. Med Sport 1962;3:1245-51.

20. Ekblom B. Applied physiology soccer. Sports Med 1986;3:50-60.

21. Whitehead ENL. Conditioning for sport. EP Publishing Co. Ltd. Yorkshire, 1975:40-2.

22. Whiters RT, Wasilewski S, Kelly L. Match analysis of Australian professional soccer players. J Hum Mov Stud 1982;8:159-76.

23. Van Gool D, Van Gerven D, Boutmans J. The physiological load imposed soccer players during real match-play. In: Reilly T, et al, editors. Science and football. London: E. \& F. Spon, 1988:51-9.

24. Reilly T. Physiological aspects of soccer. Biology of Sport 1994;11:320.

25. Mayhew SR, Wenger HA. Time-motion analysis of professional soccer. J Hum Mov Stud 1985;11:49-52.

26. Yamanaka K. Time and motion analysis in top class games. In: Reilly T, et al, editors. Science and football. London: E. \& F.N. Spon, 1988;33440

27. D’Ottavio S, Tranquilli C. La prestazione del giocatore di calcio. Sds Rivista di Cultura Sportiva Italiana 1993;24:74-8.

28. Reilly T, Thomas V. A motion analysis of work-rate in different positional roles in professional football match-play. J Hum Mov Stud 1976; 2:87-97.

29. Ohashi J, Togari H, Isokawa M, Suzuki S. Measuring movement speeds and distances covered during soccer match-play. In: Reilly T, et al, editors. Science and Football. London: E. \& F. Spon, 1988:329-33.

30. Bangsbo J, Norregaard L, Thorsoe F. Activity profile of competition soccer. Can J Sports Sci 1991;16:110-6.

31. Saltin B. Metabolic fundamentals in exercise. Med Sci Sports 1973;5: 137-46.

32. Brooks GA. Lactate production during exercise: oxidizable substrate versus fatigue agent. In: Macleod D, Maughan R, Nimmo M, Reilly T, Williams TC, editors. Exercise, benefits, limits and adaptations. London, New York: E. \& F.N. Spon, 1987:144-58.

33. Nordheim K, Vollestad NK. Glycogen and lactate metabolism during low intensity exercise in man. Acta Physiol Scand 1990;139:475-84.

34. Boobis LH. Metabolic aspects of fatigue during sprinting. In: Macleod D, Maughan R, Nimmo M, Reilly T, Williams TC, editors. Exercise, benefits, limits and adaptations. London, New York: E. \& FN Spon, 1987 : 116-43.

35. Rowell LB, Kraning KK, Evans TO, Kennedy JM, Blackmon JR, Kusumi F. Splanchnic removal of lactate and pyruvate during prolonged exercise in man. J Appl Physiol 1966;21:1773-83.

36. Kreisberg RA, Pennington LF, Bashell BR. Lactate turnover and gluconeogenesis in normal and obese humans. Effects of starvation. Diabetes 1970;19:53-5.

37. Hermansen L, Stensvold I. Production and removal of lactate during exercise in man. Acta Physiol Scand 1972;86:191-201.

38. Freund H, Gendry P. Lactate kinetics after short strenous exercise in man. Eur J Appl Physiol 1978;39:123-35.

39. Brooks GA. Anaerobic threshold: review of the concept and directions for future research. Med Sci Sports Exerc 1985;17:22-31.

40. Karlsson J, Nordesjo LO, Jorfeldt L, Saltin B. Muscle lactate, ATP and $\mathrm{CP}$ levels during exercise after physical training in man. J Appl Physiol 1972;33:199-203.

41. Knuttgen HG, Saltin B. Muscle metabolites and oxygen uptake in shortterm submaximal exercise in man. J Appl Physiol 1972;32:690-4.

42. Tesch PA, Daniels WL, Sharp S. Lactate accumulation in muscle and blood during submaximal exercise. Acta Physiol Scand 1982;114:441-6. 
43. Jacobs I, Kaijser P. Lactate in blood, mixed skeletal muscle, and FT or ST fibres during cycle exercise in man. Acta Physiol Scand 1982;114: 461-6.

44. Green HJ, Hughson RL, Orr GW, Ranney DA. Anaerobic threshold, blood lactate and muscle metabolites in progressive exercise. J Appl Physiol 1983;54:1032-8.

45. Chwalbinska-Moneta J, Robergs RA, Costill DL, Fink WJ. Threshold for muscle lactate accumulation during progressive exercise. J Appl Physiol 1989;66:2710-6.

46. Rhode HC, Espersen T. Work intensity during soccer training and matchplay. In: Reilly T, Lees A, Davids K, Murphy WJ, editors. Science and football. London, New York: E. \& FN. Spon, 1988:68-75.

47. Gerish G, Rutemoller E, Weber K. Sportsmedical measurements of performance in soccer. In: Reilly T, et al, editors. Science and football. London: E. \& F. Spon, 1988:60-7.

48. Hargreaves M. Carbohydrate and lipid requeriments of soccer. J Sports Sciences 1994;12:S13-S16.

49. Fox EL, Mathews DK. Bases fisiológicas da educação física e dos desportos. $3^{a}$ ed. Rio de Janeiro: Interamericana, 1983:p. 487.

50. Reilly T. Football. In: Reilly T, et al, editors. Physiology of sports. Londres: E. \& F.N. Spon, 1990:371-401.
51. Smaros G. Energy usage during football match In: Vecchiet L, editor. Proceedings, $1^{\text {st }}$ International Congress on Sports Medicine Applied Football. Vol. 11. D. Guanello, Rome, 1980:795-801.

52. Hochachka PW. Fuels and pathways as designed systems for support of muscle work. J Exp Biol 1985;115:149-64.

53. Banister EW, Cameron BJC. Exercise-induced hyperammonemia: peripheral and central effects. Int J Sports Med 1990;11:S129-S142.

54. Connett RJ, Honig CR, Gayeski TE, Brooks GA. Defining hypoxia: a systems view of $\mathrm{VO}_{2}$, glycolysis, energetics and intracellular $\mathrm{pO}_{2}$. J Appl Physiol 1990;68:833-42.

55. Fitts RH. Cellular mechanisms of muscle fatigue. Physiol Rev 1994;74: 49-94.

56. Zelenka V, Seliger V, Ondrej O. Specific function testing of young football players. J Sports Med 1967;7:143-7.

57. Ohashi J, Togari H, Takii T. The distance covered during matchs of the World Class soccer players (in Japanese). Proceedings of the Department of Sports Science, College of Arts and Sciences University of Tokio, 1991;25:1-5.

58. Knowles JE, Brooke JD. A movement analysis of player behaviour in soccer match performance. Proceedings of $8^{\text {th }}$ Annual Conference, British Society of Sports Physiology (Salford), 1974. 